\title{
3D-Printing Soft sEMG Sensing Structures
}

\author{
Gerjan Wolterink ${ }^{1}$, Remco Sanders ${ }^{1}$, Frodo Muijzer ${ }^{2}$, Bert-Jan van Beijnum ${ }^{3}$, Gijs Krijnen ${ }^{1}$ \\ ${ }^{1}$ Robotics And Mechatronics group, University of Twente, Enschede, The Netherlands \\ 2 Twente Medical Systems International B.V. Oldenzaal, The Netherlands \\ ${ }^{3}$ Biomedical Signal and Systems, University of Twente, Enschede, The Netherlands \\ Email: gerjan.wolterink@utwente.nl
}

\begin{abstract}
This paper describes the development and characterization of soft and flexible 3D-printed sEMG electrodes. The electrodes are printed in one go on a low cost consumer multi-material FDM printer. The printed structures do not need any further production steps to give them conductive properties. Comparison between the gold standard Ag/AgCl gel electrodes and the printed EMG electrodes with a comparable contact area show that there is no significant difference in the EMG signal amplitude. The sensors are capable of distinguishing several levels of muscle activity of the biceps brachii. This all gives 3Dprinted sEMG electrodes a high potential for creating personalized sensing structures, e.g. in prosthetic and orthotic contexts.
\end{abstract}

Keywords - 3D-Printing, Conductive, Flexible, Soft, EMG, Electrodes

\section{INTRODUCTION}

Electromyography (EMG) is a method to measure the electrical activity associated with muscle contractions. EMG gives useful information about the activity of individual muscles and is therefore widely used for the control of hand and arm assistive devices and prosthesis [1]-[6]. An example of EMG control is the Pisa/IIT SoftHand, an EMG driven soft robotic hand [7]. Nowadays surface EMG (sEMG) electrodes are only available in fixed shapes. Some electrode structures, are flexible but cannot be stretched in such a way that complex body shapes are completely covered by the electrodes. Therefore it is often still needed to place each electrode individually. Additive manufacturing, better known as 3D-printing, is a technique that stacks layers of materials to create physical three dimensional objects. 3D printing technologies enable the creation of personalised sensing structures that require minimal assembly and are easily scalable in the number of electrodes. These structures will be soft and follow body contours so they do not influence musculoskeletal deformations, greatly enhancing the users comfort and aesthetics of the devices.

In order to create sEMG sensing structures a conductive material needs to be embedded in a 3D-printed structure. This can be done using structures containing channels and/or cavities and filling these with a conductive material. These structures can be printed with a wide variety of printing techniques. However this method requires extra fabrication steps and adds limitations to the sensor design. Fused deposition modelling (FDM) is a technique where a 3D-model is built up

This works was developed within the SoftPro project, funded by the European Union's Horizon 2020 Research and Innovation Programme under Grant Agreement No. 688857 by melting a thermoplastic filament and depositing the material layer by layer. The filament is usually made from stiff materials such as polylactic acid (PLA), acrylonitrile butadiene styrene (ABS). Other materials are i.e. thermoplastic polyurethanes (TPU) [8], these materials are flexible and elastic. To create conductive prints these filaments are mixed with conductive particles made out of copper or carbon [9]-[11]. To date, attempts to create 3D-printed biopotential electrodes are limited to rigid single material designs [12]. With the use of multimaterial printers soft flexible structures with conductive and insulating materials can be printed in one go as shown in Fig. 1. Currently developments are made in embedding electronics inside a flexible sensing structure which is designed to fit an individual user.
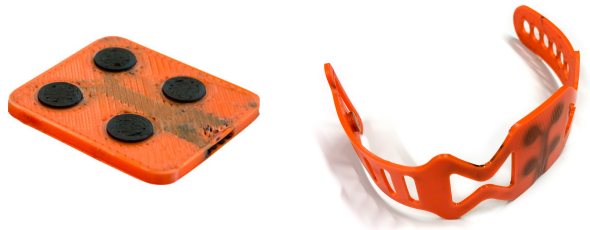

Fig. 1. Photograph of 3D-Printed electrodes using flexible conductive filaments.

\section{Methodology}

\section{A. Electrode fabrication}

The sensing structures are designed using Fusion 360 (Autodesk, USA), Fig. 2 shows an assembled sensing structure. The dark parts are printed with conductive TPU (PI-ETPU, Palmiga Innovation, Sweden) and have a diameter of $16 \mathrm{~mm}$. The transparent parts are made of non-conductive TPU (NinjaFlex, Fenner Drives, USA) and are used as insulation and to keep the electrodes at equal distance. The distance from centre to centre of the electrodes is $20 \mathrm{~mm}$. The structures are printed on a FlashForge Creator Pro (FlashForge Corporation, China) fitted with the Flexion Extruder (Diabase Engineering, USA) and the cost of the raw materials used is estimated to be around $0.20 €$. A personal computer running slicer software (Simplify3D, Inc., USA) slices the model and manages the control of the printer. The structures are printed with a $150 \mu \mathrm{m}$ layer height. The nozzle diameter used for the PI-ETPU conductive TPU is $800 \mu \mathrm{m}$ and for the dielectric NinjaFlex TPU $600 \mu \mathrm{m}$. Connections to the electrodes are made by melting in a stranded wire on the connection pads. Shrink tubing is used for insulation and strain relief. The $20 \mathrm{~mm}$ long unshielded wire is connected to a standard header pin connector that interfaces with the shielded wires of a biopotential amplifier. 

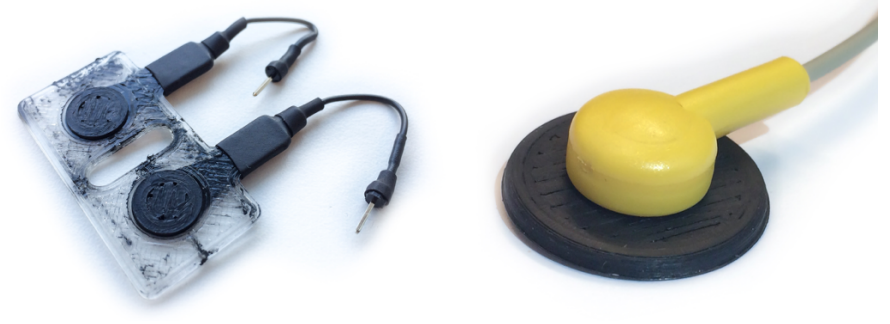

Fig. 2. Left: 3D-printed electrode after assembly. Right: Connected printed electrode used for patient ground.

\section{B. Protocol}

Six healthy adult (18+) volunteers enroled in the measurement, approved by the faculty ethics committee. Prior to application of the electrodes, the skin around the biceps brachii is prepared according to SENIAM recommendations [13], [14]. If hair is present it is shaved. Next the skin and the electrodes are cleaned with alcohol. After the alcohol is evaporated the electrodes are placed according to the SENIAM recommendations above the biceps brachii. The patient ground electrode is a disk with a diameter of $25 \mathrm{~mm}$ made out of PI-ETPU and is placed on the subjects wrist on the opposite arm. Prior to the application, the patient ground electrode is moistened with use of a cotton swab dipped in tap water. The printed sensing structures are placed next to $\mathrm{Ag} / \mathrm{AgCl}$ gel $(\mathrm{AgCl})$ electrodes (Covidien, Ireland). The $\mathrm{AgCl}$ electrodes are placed next to each other resulting in an inter electrode distance of $20 \mathrm{~mm}$. The cross-section of the electrolyte is $16 \mathrm{~mm}$. The electrodes are connected to a TMSi Refa (Twente Medical Systems International B.V., The Netherlands). The Refa is connected to a computer via a bidirectional optical fiber. The EMG data is sampled at $1250 \mathrm{~Hz}$ and is visualised and stored using the TMSi Matlab Interface.

During all the measurements the subject remains seated. For the first measurement the subject is asked to perform 3 short isometric contractions. The voluntary contraction is measured by performing elbow flexion followed by extension without any load (concentric contraction). This movement is repeated 3 times as well.

The second measurement, the load test, the subject is asked to place his elbow on an arm rest and to hold a handle that is connected to a load, see Figure 3. The measurement starts with the subject keeping this position with no load on the handle. After 10 seconds the load is increased with $1 \mathrm{~kg}$, the subject has to keep her/his arm in position causing the biceps to contract. Every 10 seconds the load is increased with $1 \mathrm{~kg}$ till the total load amounts to $5 \mathrm{~kg}$.

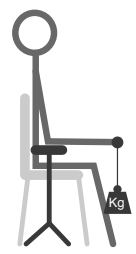

Fig. 3. Position of the subject performing the load experiment. The elbow is placed on an arm rest, the load is increased form $0 \mathrm{~kg}$ to $5 \mathrm{~kg}$ ever 10 seconds.

\section{Signal analysis}

The data obtained from the measurements is loaded into Matlab and further processed. First drift and movement artefacts are removed form the signal with the use of a $20 \mathrm{~Hz}$ high pass filter (3rd order Butterworth). The mains noise is filtered out using a $50 \mathrm{~Hz}$ notch filter. The higher frequencies are filtered out using a $250 \mathrm{~Hz}$ low pass filter (3rd order Butterworth). Finally the signal envelope is taken by using a moving RMS window of 25 samples $(20 \mathrm{~ms})$. The data of the load test is segmented into windows of 5 seconds, each representing a load ranging from $0 \mathrm{~kg}$ to $5 \mathrm{~kg}$.

\section{Statistical analysis}

To prove, or reject, the difference between $\mathrm{AgCl}$ and TPU electrodes, the mean envelope per electrode is taken per subject of the $5 \mathrm{~kg}$ sample. Next the values of the $\mathrm{AgCl}$ and the TPU electrode are tested using a paired t-test. The increase of muscle activity is proven by taking the mean envelope per subject, per load of one electrode and test this against the values of the next load using a paired t-test.

\section{RESULTS}

Fig. 4 shows the time domain data of the printed and $\mathrm{AgCl}$ electrodes for a given subject performing three isometric contractions followed by three concentric contractions. The figure suggest that the signal amplitudes are highly correlated.

The mean envelope and corresponding standard error per subject per electrode are visualized in Fig. 5. For all subjects, except subject 3, the mean envelope of the $\mathrm{AgCl}$ is significant $(p<0.01)$ higher. For subject 3 the printed electrodes mean envelope was significant higher $(p<0.01)$. The mean envelope over all subjects of the $5 \mathrm{~kg}$ load experiment shown in Fig. 6 seems to indicate that the $\mathrm{AgCl}$ electrode has a higher amplitude. However no significant difference $(p=0.10)$ in the amplitude between the $\mathrm{AgCl}$ and TPU electrodes is found. The visually observed difference can be explained since the electrodes are placed next to each other above the biceps brachii and are therefore not measuring the exact same volume.

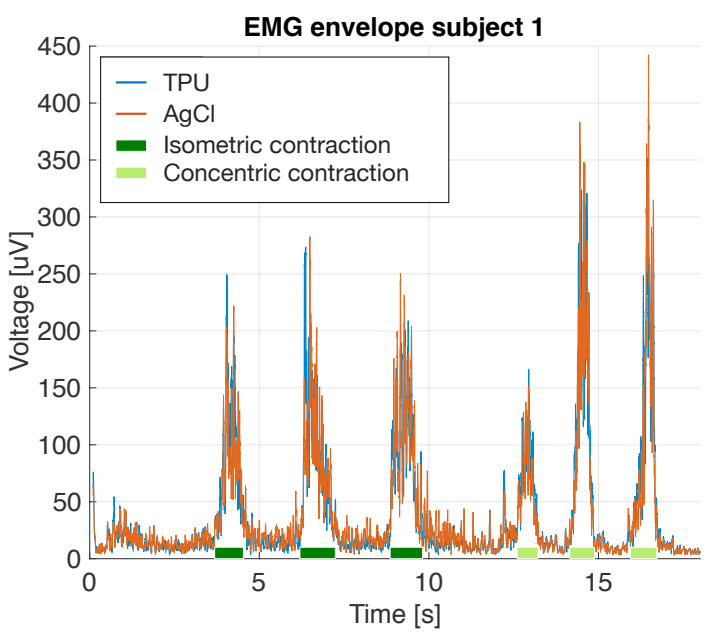

Fig. 4. Envelope of the measured EMG with the printed and $\mathrm{Ag} / \mathrm{AgCl}$ gel electrodes for a given subject performing three isometric contractions followed by three concentric contractions. 


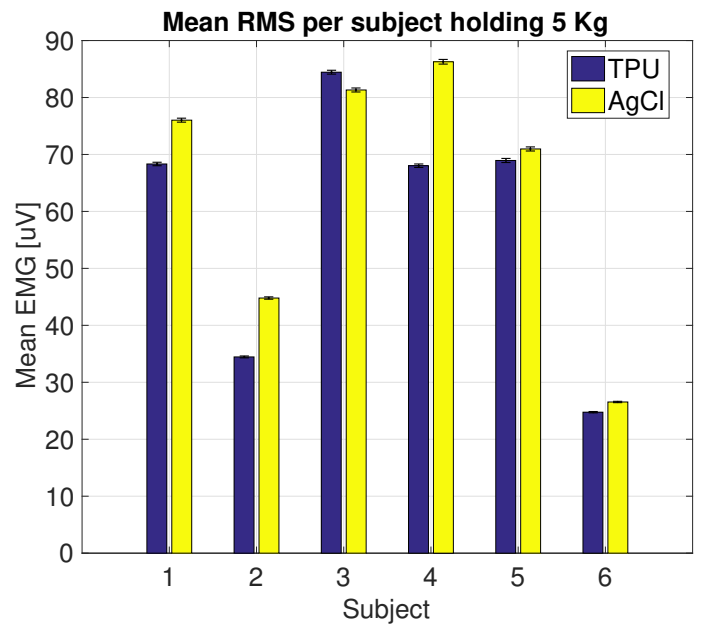

Fig. 5. Mean envelope value and standard error of all subjects performing the $5 \mathrm{~kg}$ load test.

Another noteworthy observation is the difference between subjects mean envelope as indicated in Fig. 5.

The mean envelope over all subjects per weight are visualized in Fig. 6. The mean envelope value for the $\mathrm{AgCl}$ and TPU electrodes increases significant when the load is increased (Table I).

TABLE I. DIFFERENCES IN THE MEAN OF MEASURED MUSCLE ACTIVITY BETWEEN TWO LOADS AND CORRESPONDING $p$-VALUE.

\begin{tabular}{llllll}
\hline Between weights $[\mathrm{kg}]$ & $\mathbf{0 - 1}$ & $\mathbf{1 - 2}$ & $\mathbf{2 - 3}$ & $\mathbf{3 - 4}$ & $\mathbf{4 - 5}$ \\
\hline Difference AgCl $[\mu \mathrm{V}]$ & 5.52 & 5.99 & 6.15 & 10.28 & 15.60 \\
P-value & 0.062 & 0.007 & 0.043 & 0.007 & 0.021 \\
\hline Difference TPU $[\mu \mathrm{V}]$ & 6.10 & 6.27 & 5.61 & 7.82 & 15.06 \\
P-value & 0.046 & 0.009 & 0.033 & 0.002 & 0.008 \\
\hline
\end{tabular}

\section{CONCLUSION}

Flexible sEMG electrodes are successfully printed on a low cost consumer FDM 3D-printer, with costs well under one euro. Comparison between the gold standard $\mathrm{Ag} / \mathrm{AgCl}$ gel electrodes and the printed EMG electrodes with a comparable

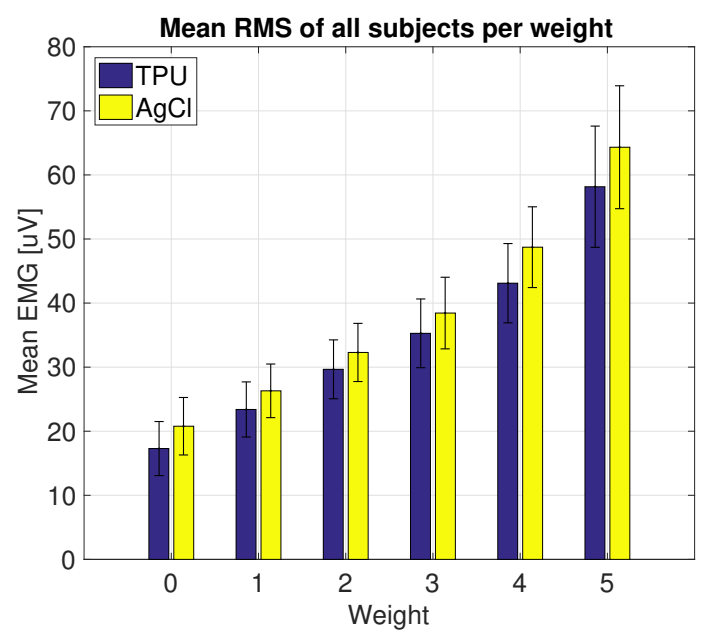

contact area show that there is no significant difference in the EMG signal amplitude. Both electrodes are perfectly capable of distinguishing several levels of muscle activity of the biceps brachii.

3D-printed sEMG electrodes have a high potential in practical applications. The presented structures are relatively simple. But 3D-printing enables fast and cheap creation of far more complex structures that are personalised to the user. Complex wire routing and shielding can be done in three dimensions inside of the structures, strongly reducing the amount of wiring needed. In the future sEMG electrodes might be integrated in assistive devices and prosthesis that are printed in one go.

\section{REFERENCES}

[1] D. Staudenmann, K. Roeleveld, D. F. Stegeman, and J. H. van Dieen, "Methodological aspects of semg recordings for force estimation-a tutorial and review," J Electromyogr Kinesiol, vol. 20, no. 3, pp. 375-87, 2010. [Online]. Available: http://www.ncbi.nlm.nih.gov/ pubmed/19758823

[2] R. Merletti, A. Botter, A. Troiano, E. Merlo, and M. A. Minetto, "Technology and instrumentation for detection and conditioning of the surface electromyographic signal: state of the art," Clin Biomech (Bristol, Avon), vol. 24, no. 2, pp. 122-34, 2009. [Online]. Available: http://www.ncbi.nlm.nih.gov/pubmed/19042063

[3] M. B. Reaz, M. S. Hussain, and F. Mohd-Yasin, "Techniques of emg signal analysis: detection, processing, classification and applications (correction)," Biol Proced Online, vol. 8, p. 163, 2006. [Online]. Available: http://www.ncbi.nlm.nih.gov/pubmed/19565309

[4] M. Piotrkiewicz and B. Kuraszkiewicz, "Afterhyperpolarization of human motoneurons firing double and triple discharges," Front Hum Neurosci, vol. 8, p. 373, 2014. [Online]. Available: http: //www.ncbi.nlm.nih.gov/pubmed/24910608

[5] R. G. Scalisi, M. Paleari, A. Favetto, M. Stoppa, P. Ariano, P. Pandolfi, and A. Chiolerio, "Inkjet printed flexible electrodes for surface electromyography," Organic Electronics, vol. 18, pp. 89-94, 2015. [Online]. Available: 〈GotoISI $\rangle: / /$ WOS:000349548400013

[6] A. E. Schultz and T. A. Kuiken, "Neural interfaces for control of upper limb prostheses: the state of the art and future possibilities," $P M \& R$, vol. 3, no. 1, pp. 55-67, 2011.

[7] S. Godfrey, A. Ajoudani, M. Catalano, G. Grioli, and A. Bicchi, "A synergy-driven approach to a myoelectric hand," in Rehabilitation Robotics (ICORR), 2013 IEEE International Conference on. IEEE, 2013, pp. 1-6.

[8] Fenner Drives. Ninjatek.com. Url: https://ninjatek.com, Accessed: 31-01-2017. [Online]. Available: https://ninjatek.com

[9] Multi3D. Electrifi Conductive 3D Printing Filament. Https://www.multi3dllc.com/product/electrifi-3d-printing-filament/, Accessed: 13-06-2017. [Online]. Available: https://www.multi3dllc.com/ product/electrifi-3d-printing-filament/

[10] ProtoPlant, makers of Proto-pasta. Composite PLA - Electrically Conductive Graphite. Url: https://www.proto-pasta.com/, Accessed: 31-01-2017. [Online]. Available: https://www.proto-pasta.com/

[11] Palmiga Innovation. Material info for PI-ETPU 95-250 Carbon Black the conductive and flexible 3D printing filament. Url: http://rubber3dprinting.com/pi-etpu-95-250-carbon-black/, Accessed: 26-05-2017. [Online]. Available: http://rubber3dprinting.com/ pi-etpu-95-250-carbon-black/

[12] S. Krachunov and A. J. Casson, "3d printed dry eeg electrodes," Sensors, vol. 16, no. 10, p. 1635, 2016.

[13] H. J. Hermens, B. Freriks, C. Disselhorst-Klug, and G. Rau, "Development of recommendations for semg sensors and sensor placement procedures," J Electromyogr Kinesiol, vol. 10, no. 5, pp. 361-74, 2000. [Online]. Available: http://www.ncbi.nlm.nih.gov/ pubmed/11018445

[14] SENIAM. Url: http://seniam.org, Accessed: 23-03-2017. [Online]. Available: www.seniam.org

Fig. 6. Mean envelope and standard error of all subject per load 